\title{
Attenuation of Docetaxel-induced Oxidative Stress and Apoptosis in HEK 293 Human Embryonic Kidney Cells by Curcumin Treatment
}

\author{
Suleyman $\operatorname{Ilhan}^{1 *}$ (D) \\ ${ }^{1}$ Manisa Celal Bayar University, Faculty of Science and Letters, Biology Department, Manisa, Turkey \\ *suleyman.ilhan@cbu.edu.tr \\ * Orcid: 0000-0002-6584-3979
}

Received: 13June 2020

Accepted: 20 February 2021

DOI: $10.18466 /$ cbayarfbe. 752495

\begin{abstract}
Docetaxel (DOC) is a chemotherapeutic that induces microtubule stabilization. It is often used in breast, prostate, lung and gastric cancers but severe side effects such as cardiotoxicity, neurotoxicity, hepatotoxicity, and nephrotoxicity limit its usage. Curcumin (CUR), a natural bioactive compound derived from turmeric. Here, the possible preventive effect of CUR against DOC-induced oxidative stress and apoptosis on HEK-293 immortalized human embryonic kidney cells. Viability was assessed via MTT assay. The generation of ROS was measured by CM-H2DCFDA dye. Phosphatidylserine externalization and caspase $3 / 7$ activity were used to measure apoptosis. CUR pretreatment remarkably inhibited DOCinduced cell viability reduction, ROS generation, and cell apoptosis in HEK-293 cells. Moreover, this study revealed that CUR pretreatment decreased caspase-3 activity. Thus, this study highlights the novel pharmacological mechanisms of CUR and understanding the detailed mechanisms of CUR action.
\end{abstract}

Keywords: Apoptosis, curcumin, docetaxel, HEK-293, oxidative stress.

\section{Introduction}

Despite the novel technological and scientific developments, cancer is still one of the biggest threats to humanity for decades. Many strategies are being done to struggle with cancer [1]. Among them, chemotherapy is the most effective one and considered the backbone of the treatment. However, serious side-effects in normal tissues such as cardiotoxicity, neurotoxicity, hepatotoxicity, and nephrotoxicity limit their usages [25]. Thus, there is an instant need to find new adjuvant compounds to diminish the chemotherapy-induced severe side effects.

Taxanes that promote microtubule stabilization are critical chemotherapy drugs in the treatment of a wide range of solid tumors [6-9]. Of taxanes, Docetaxel (DOC) is often used in breast, lung, prostate and gastric cancers, but its frequent use often leads to nephrotoxicity, especially in lung cancer patients [10]. Chronic nephrotoxicity of anticancer drugs may have multiple causes and are mediated by various mechanisms. In this study, the effects of DOC were investigated on human kidney embryonic cells (HEK293) which could represent the possible target tissue and widely used in in vitro toxicology studies. One of the most important mechanisms is reactive oxygen species
(ROS) formation and subsequently induction of hepatocyte apoptosis [11]. The induction of ROS also decreases endogenous antioxidants via activation of caspases. To avoid these harmful effects of oxidative stress, it is essential to increase the cell's antioxidant defenses with natural compounds having antioxidant activity.

Curcumin (CUR), known as diferuloylmethane, is originated from Curcuma longa rhizome. It has been shown to have several biological activities [12-15]. CUR is a potent oxygen free radical scavenger and can inhibit the production of ROS and prevent oxidative stress both in vitro and in vivo [16]. Previous studies demonstrated that CUR treatment can ameliorate nephrotoxicity by preventing oxidative stress caused by cisplatin, gentamicin and cyclosporine and paracetamol in rats [17-19].

Taken together, the current study was aimed to investigate the protective effect of the CUR against DOC-induced oxidative stress and apoptotic cell death on HEK-293 immortalized human embryonic kidney cells. 


\section{Materials and Methods}

\subsection{Materials}

CUR was provided by Sigma Chemical Co (USA). To prepare a stock solution, CUR was dissolved in 1 $\mathrm{mg} / \mathrm{mL}$ ethanol $(\mathrm{EtOH})$ and stored at $4^{\circ} \mathrm{C}$. DOC was purchased from Sigma Chemical Co and dissolved in dimethyl sulfoxide (DMSO). The EtOH and DMSO concentrations used in this analysis were lower than $0.1 \%$ and were not cytotoxic. All other chemicals were obtained from Sigma.

\subsection{Cell culture conditions}

The human embryonic kidney cell line HEK-293 (CRL11268) was purchased from ATCC (American Type Culture Collection, USA). The cells were cultured by using Eagle's Minimum Essential Medium including $10 \%$ fetal bovine serum and $1 \%$ penicillin/streptomycin in $75 \mathrm{~cm}^{2}$ polystyrene cell culture flasks (Cellstar, UK). Cell culture was maintained in a standard incubator containing $5 \% \mathrm{CO}_{2}$ at $37^{\circ} \mathrm{C}$.

\subsection{Cell viability and morphology}

MTT assay (3-(4,5-dimethylthiazol-2-yl)-2,5diphenyltetrazolium bromide) was conducted to evaluate cell viability. Disaggregation of cells was carried out with trypsin/EDTA. A total of $10^{4}$ cells were propagated onto 96-well plates in $100 \mu \mathrm{L}$ medium per well. After $24 \mathrm{~h}$ cell attachment, cells were exposed to increasing concentrations of DOC, CUR, or sequentially. MTT $(20 \mu \mathrm{L})$ was added at 24,48 and $72 \mathrm{~h}$ time points. The dye was carefully drained and $200 \mu \mathrm{L}$ DMSO was added to dissolve formed formazan crystals. The optic densities were recorded by a microplate reader at $570 \mathrm{~nm}$ (Tecan Infinite 200 PRO, Switzerland) [20]. Olympus IX53 inverted microscope was used to monitor HEK-293 cell morphology after DOC, CUR or sequential treatment (Tokyo, Japan).

\subsection{Analysis of ROS generation}

The formation of ROS in HEK-293 cells was measured by cell-permeable indicator dye [5-(and-6)chloromethyl-2,7-dichlorodihydrofluorescein diacetate acetyl ester dye (CM-H ${ }_{2}$ DCFDA, MW 577.8 Da, Molecular Probes, Eugene, OR)]. The $\mathrm{CM}-\mathrm{H}_{2} \mathrm{DCFDA}$ passively enters the cells and remains non-fluorescent until oxidation occurs in the cell. After oxidation, it fluoresces in the cell. The cell suspension was $\left(10^{5}\right.$ cells $/ \mathrm{mL}$ ) transferred on 96-wells in $200 \mu \mathrm{L}$ medium containing $\mathrm{CM}-\mathrm{H}_{2} \mathrm{DCFDA}$ dye. The plate was then incubated for $45 \mathrm{~min}$. at $37^{\circ} \mathrm{C}$. As a control, cells were also exposed to $10 \mathrm{mM} \mathrm{N}$-acetylcysteine (NAC) which is a ROS scavenger. The amount of fluorescence was then measured at $485-520 \mathrm{~nm}$ by a microplate reader (Tecan Infinite 200 PRO, Switzerland) and also observed with fluorescence microscopy (Olympus, Tokyo, Japan).

\subsection{Flow cytometric analysis of apoptosis}

For analyzing apoptosis, phosphatidylserine externalization was detected via Muse ${ }^{\mathrm{TM}}$ Annexin V and Dead Cell kit (Millipore, USA). HEK-293 cells in a density of $4 \times 10^{5}$ were seeded in $2 \mathrm{~mL}$ medium and incubated $24 \mathrm{~h}$ for attachment. Then, attached cells were exposed to compounds alone or sequentially for $48 \mathrm{~h}$. After incubation, centrifugation was performed at $1000 \times \mathrm{g}$ for $10 \mathrm{~min}$ and supernatants were removed. The remaining pellets were suspended with a culture medium. Equal amounts of cell suspension and Muse ${ }^{\mathrm{TM}}$ Annexin-V \& Dead Cell kit solution were mixed and incubated for $20 \mathrm{~min}$ in the dark. The analysis was performed using Muse ${ }^{\mathrm{TM}}$ Cell Analyzer (Millipore, USA). For each condition at least 10,000 cells were analyzed.

\subsection{Caspase $3 / 7$ activity assay}

Caspase-Glo 3/7 Assay (Promega, USA). Cells were $\left(10^{4}\right.$ cells per well) treated with the desired concentrations of the compounds. After incubation periods, CaspaseGlo 3/7 reagent $(100 \mu \mathrm{L})$ was added and incubated for $1 \mathrm{~h}$ in the dark. The formed luminescence was detected by a microplate reader (Tecan Infinite 200 PRO, Switzerland).

\subsection{Statistical Analysis}

Each experiment was done in duplicate, and three experiments were performed for each condition. Results were presented as mean $\pm \mathrm{SD}$. The Student's t-test was performed to analyze the differences between the two groups. For analysis of three or more groups, the first one-way analysis of variance test was done and then Dunnett's t-test was performed. The half-maximal inhibitory concentration ( $\mathrm{IC}_{50}$ ) was calculated via Graph Pad Prism 5.0 (San Diego, USA). A p-value of $<0.05$ was considered statistically significant.

\section{Results and Discussion \\ 3.1. Preventive effect of CUR on DOC-induced cytotoxicity in HEK-293 cells}

Today, CUR has attracted intense interest in the protection of normal tissues from chemotherapyinduced toxicity. Before determining the preventive effect of CUR on DOC-induced cytotoxicity in HEK293 cells, first, the effects of single CUR or DOC on the viability of cells were investigated. For this aim, HEK293 cells were exposed to increasing concentrations of CUR $(5-100 \mu \mathrm{g} / \mathrm{mL})$ or DOC $(0.1-1000 \mathrm{nM})$ for different time points and viability was assessed via MTT assay. As seen in Fig. 1, the increasing concentrations of single CUR or DOC induced a time- 
dependent reduction in cell viability $(\mathrm{p}<0.05)$. The $\mathrm{IC}_{50}$ value of CUR calculated from cell viability data was found to be $12.5 \pm 0.52 \mu \mathrm{g} / \mathrm{mL}$ at $48 \mathrm{~h}(\mathrm{p}<0.05)$. The cell viability was $91 \%$ at $5 \mu \mathrm{g} / \mathrm{mL}$ CUR concentration but significantly decreased by $25 \mu \mathrm{g} / \mathrm{mL}$ CUR concentration (17\% viability) at $48 \mathrm{~h}$. Therefore, 5, 10 and $25 \mu \mathrm{g} / \mathrm{mL}$ concentrations were chosen as a low-, medium- and high-concentration groups, respectively. There are limited studies demonstrating the effects of CUR on HEK-293 cells in the literature. Concentrationdependent inhibition in cell viability by different incubation times was reported for HEK-293 cells and calculated $\mathrm{IC}_{50}$ values were quite different in these studies. Rao et al. calculated the $\mathrm{IC}_{50}$ value of CUR on HEK-293 cells as $5.0 \pm 0.6 \mu \mathrm{M}$ at $72 \mathrm{~h}$ [21]. In different studies, the $\mathrm{IC}_{50}$ value of CUR was calculated at $24 \mathrm{~h}$ and found to be $29.8 \mu \mathrm{M}$ and $458.14 \mu \mathrm{M}$, respectively $[22,23]$. Only Zhao et al. calculated the $\mathrm{IC}_{50}$ value of CUR at $48 \mathrm{~h}$ and found it as $11 \mu \mathrm{M}$ which was similar to the results of the current study [24]. The $\mathrm{IC}_{50}$ value of DOC was also calculated in HEK-293 cells and found to be $5.5 \pm 2.5 \mathrm{nM}$ at $48 \mathrm{~h}$. Contrary to CUR, there is no study investigating the effect of DOC on HEK-293 cells. Thus, this study is the first study revealing the effect of DOC on HEK-293 cells.

To screen the possible preventive effect of CUR on DOC-induced cytotoxic effects, cells were pretreated with 5,10 or $25 \mu \mathrm{g} / \mathrm{mL}$ CUR for $48 \mathrm{~h}$ and then treated for an additional $48 \mathrm{~h}$ with $10 \mathrm{nM}$ DOC. As demonstrated in Fig. 2, 5 and $10 \mu \mathrm{g} / \mathrm{mL}$ CUR pretreatment significantly increased cell viability compared with the $10 \mathrm{nM}$ DOC treated group $(\mathrm{p}<0.05)$. However, in the $25 \mu \mathrm{g} / \mathrm{mL}$ CUR pretreated group, cell viability decreased as compared to the $10 \mathrm{nM}$ DOC treated group $(p<0.05)$. From these data, it can be concluded that CUR pretreatment with low and medium concentrations prevented DOC-induced cytotoxicity and cell damage in HEK-293 cells. However, this effect was not observed at high concentrations of CUR.

Nephrotoxicity is a serious and well described chemotherapy-associated side-effect limiting its clinical use. Ortega-Domínguez et al. revealed that CUR treatment prevented cisplatin-induced nephrotoxicity by diminishing mitochondrial abnormalities and necrosis [25]. In vivo part of the study was also revealed that pretreatment of CUR prevented all the histological abnormalities in the kidney as compared to only the cisplatin received group. In another study, mitomycininduced kidney damage was attenuated by CUR pretreatment in mice [26]. Their results further indicate that CUR pretreatment could reduce chemotherapyinduced cytotoxicity and nephrotoxicity.

To investigate the changes in cell morphology, HEK293 cells were also analyzed by an inverted microscope.
Observations supported the cell viability results and showed that DOC treatment of HEK-293 cells led to rounding of cells indicating cell death. Moreover, loss of cell attachment to the well plates was observed, however, the number of non-adherent cells was decreased by CUR pretreatment (Figure 3).

\subsection{CUR suppresses the production of ROS in DOC- treated HEK-293 cells}

Oxidative stress and ROS accepted as the possible mechanisms responsible for DOC induced cytotoxicity and scavenging of ROS in normal tissues are known to be effective for protecting cells from chemotherapyinduced toxicity. In many studies, it was shown that CUR could inhibit cytotoxicity via diminishing cellular ROS [27, 28]. Thus, to enlighten the role of CUR in preventing DOC-induced cytotoxicity in HEK-293 cells, cellular ROS levels were measured after staining with CM-H2DCFDA and also observed with fluorescence microscopy. As shown in Fig. 4, cellular ROS was significantly induced after exposure to DOC treatment in HEK-293 cells. ROS levels were significantly diminished in CUR pretreated and NAC treated cells $(\mathrm{p}<0.05)$.

In the study of Ortega-Domínguez et al., it has been shown that CUR can inhibit ROS as strongly as NAC. In the same study, it was also revealed that CUR attenuates cisplatin-induced mitochondrial damage via diminishing ROS production [25]. CUR not only reduces ROS levels but induces cellular defense machinery such as superoxide dismutase (SOD), catalase (CAT), and glutathione peroxidase (GTP) activities [29]. Sheu et al. showed that CUR pretreatment increased the SOD activity and, in this way, enhanced the antioxidant capacity of normal tissues [30]. From these results, it can be concluded that the possible mechanism of ROS decreasing effect in HEK-293 cells pretreated with CUR might be associated with the direct free radical scavenging activity or indirect induction of antioxidant defense systems.

\subsection{The effects of CUR pretreatment on cell apoptosis in DOC-treated HEK-293 cells}

Growing evidence shows that chemotherapy-induced cytotoxicity is mediated by apoptotic induction following oxidative stress. [31]. If ROS levels excessively increase in the cell, redox imbalanced cells become more vulnerable to apoptosis. To elucidate whether the preventive effects of CUR were related to the prevention of cell apoptosis, flow cytometric analysis was done. Cells undergoing early and late apoptosis after exposure to DOC were stained by Annexin V-FITC and PI and found that DOC treatment induced apoptotic cell death in HEK-293 cells. 
A
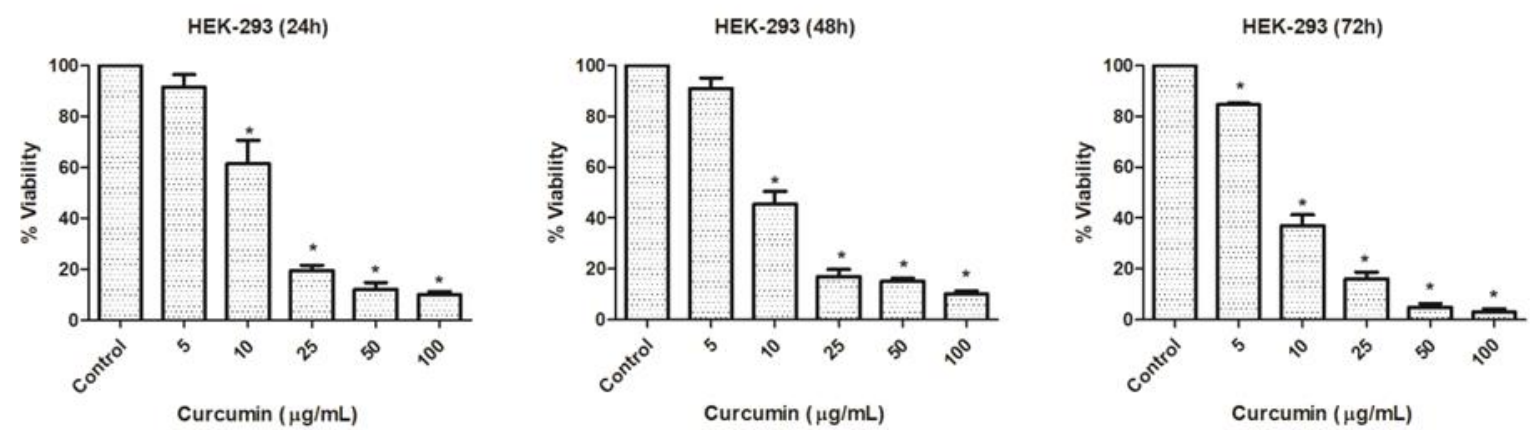

B
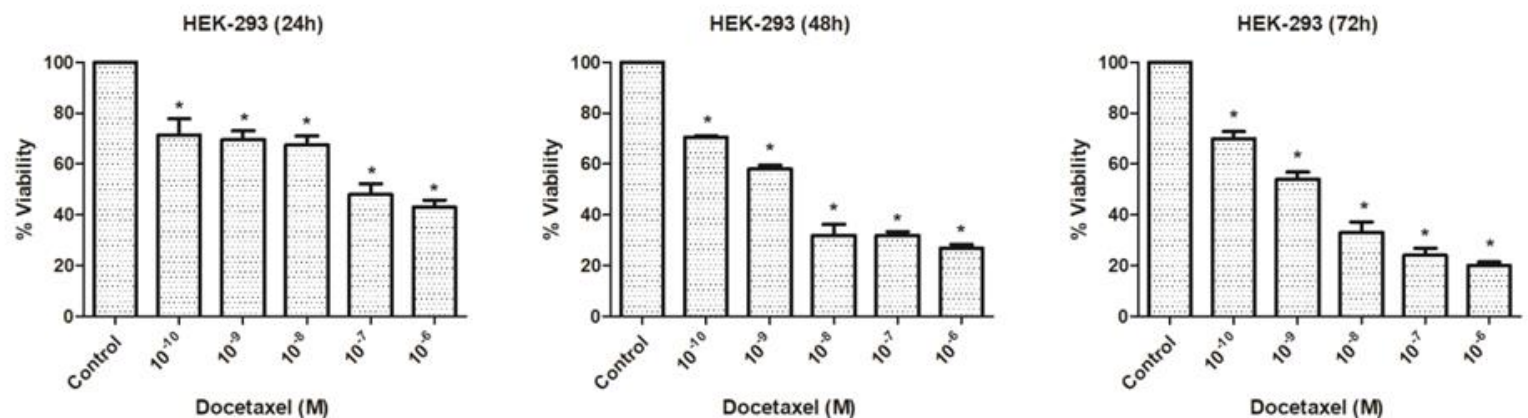

Figure 1: The effect of CUR (A) and DOC (B) on the viability of HEK-293 cells at 24, 48 and $72 \mathrm{~h}\left({ }^{*} \mathrm{p}<0.05\right.$ compared with the untreated control group).

However, the percentage of stained cells was significantly decreased in CUR pretreated group $(\mathrm{p}<0.05)$ (Figure 5).

HEK-293 (48h)

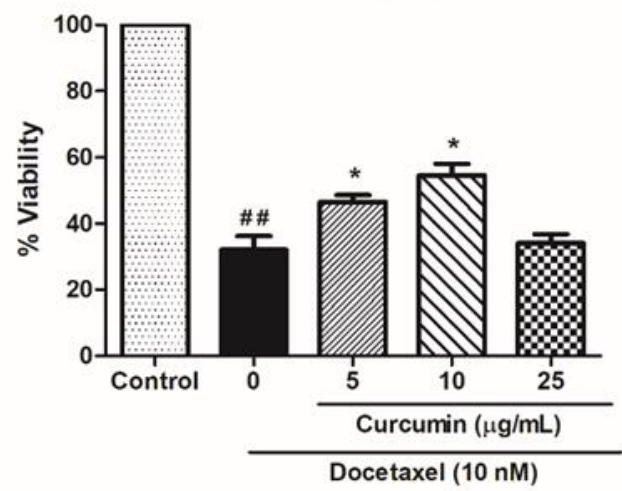

Figure 2: The effect of CUR pretreatment on the viability of HEK-293 cells under DOC exposure. Cells were treated with 5,10 or $25 \mu \mathrm{g} / \mathrm{mL}$ CUR for $48 \mathrm{~h}$ then treated with $10 \mathrm{nM}$ DOC for $48 \mathrm{~h} \quad(\# \# \mathrm{p}<0.05$ as compared to untreated control group, $* \mathrm{p}<0.05$ as compared to $10 \mathrm{nM}$ DOC treatment group).

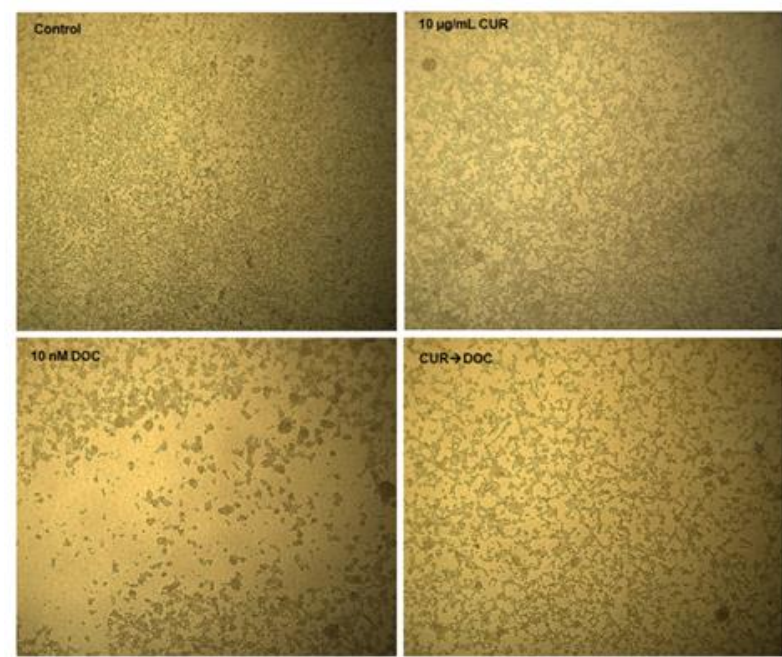

Figure 3: The effect of CUR pretreatment on the cell morphology of DOC-treated HEK-293 cells (20X).

After triggering apoptosis, the process is carried out by an enzyme family namely caspases. Among the caspase family, initiator caspases trigger effector caspases (caspases 3, 6, and 7) which are the final players that execute apoptosis [32]. 
After demonstrating the preventive effects of CUR pretreatment on apoptosis, caspase $3 / 7$ activity was performed to confirm the data. According to results, pretreatment with CUR decreased the activity as compared to single DOC treatment $(\mathrm{p}<0.05)$ (Figure 5). These observations are parallel with the previous studies that investigate the preventive effect of CUR on cell apoptosis. Benzer et al. investigated the preventive effect of CUR on doxorubicin-induced apoptosis and demonstrated that pretreatment with CUR decreased the caspase-3 activity [33]. Dai et al., demonstrated that CUR pretreatment protected cells from caspase activation and following apoptosis which was triggered by colistin [34].
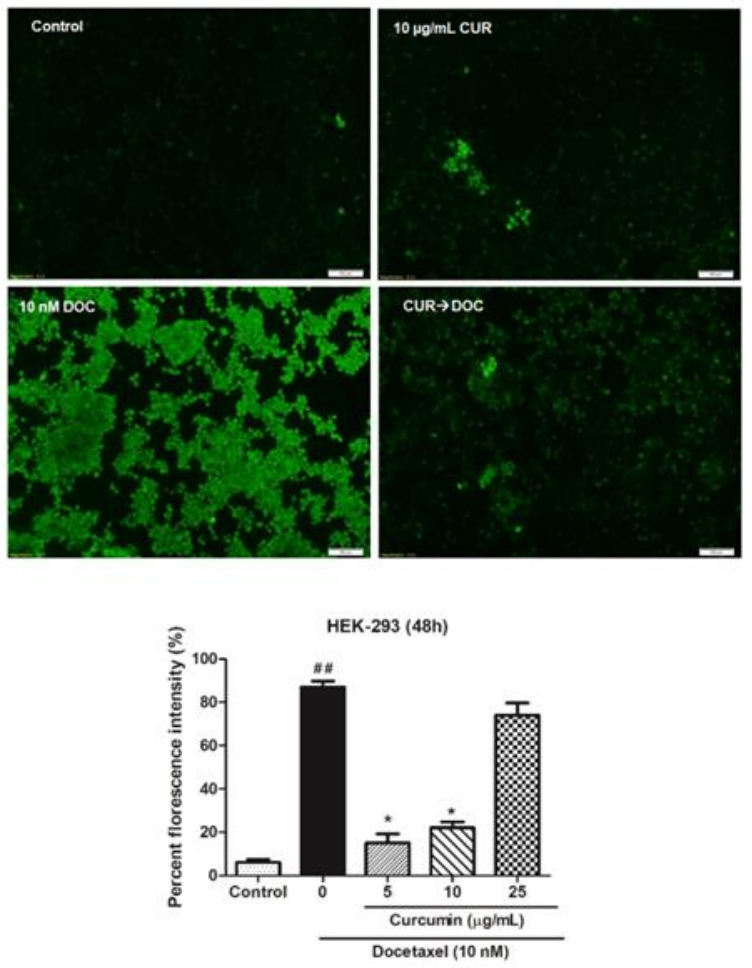

Figure 4: CUR ameliorates DOC-induced ROS in HEK-293 cells.

\section{Conclusion}

In summary, pretreatment with low concentrations of CUR can alleviate DOC-induced ROS levels in HEK293 human kidney embryonic cells. The CUR treatment also protects the cell from DOC-induced apoptotic cell death through inhibition of caspases 3 and 7 and increases the viability of cells. However, the detailed protection mechanisms of the CUR in HEK-293 cells require further investigations in vitro and in vivo. Understanding the detailed mechanisms of CUR action could lead to novel renoprotective interventions.

\section{Author's Contributions}

Süleyman İlhan: Drafted and wrote the manuscript, performed the experiment and result analysis.
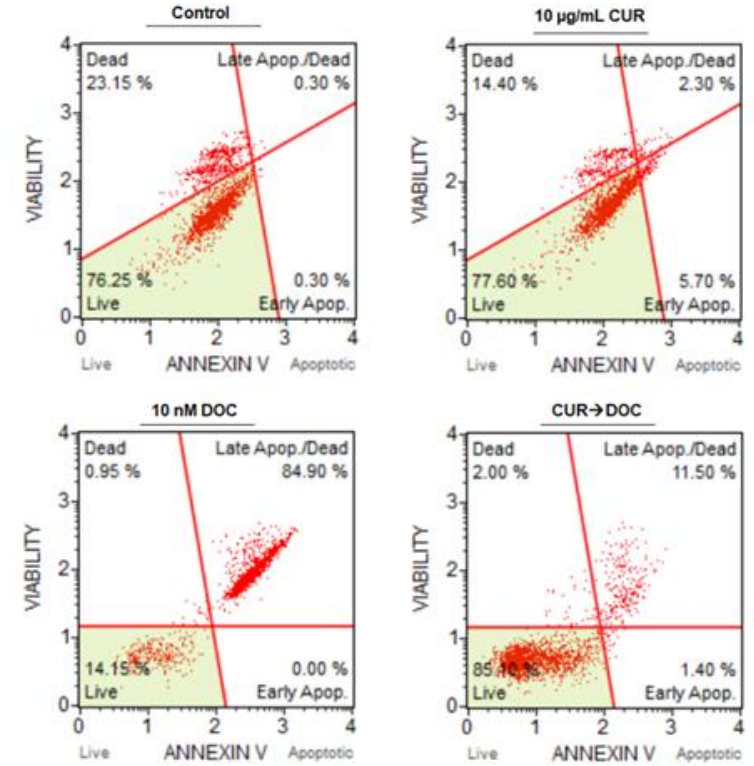

HEK-293 (48h)

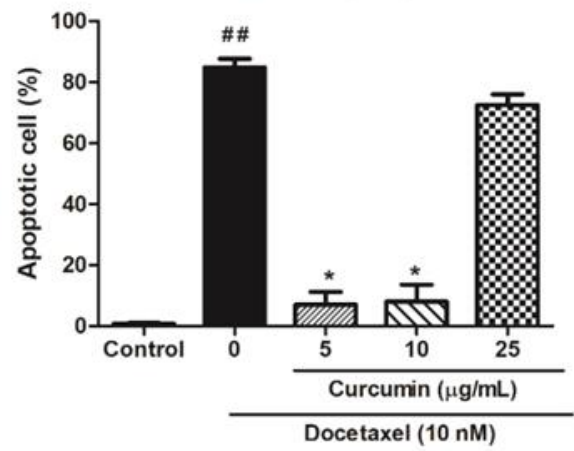

Figure 5: CUR pretreatment inhibited DOC-induced apoptosis in HEK-293 cells at $48 \mathrm{~h} \quad(\# \# \mathrm{p}<0.05$ as compared to the untreated control group, $* \mathrm{p}<0.05$ as compared to $10 \mathrm{nM}$ DOC treatment group).

\section{Ethics}

There are no ethical issues after the publication of this manuscript.

\section{References}

1. Bray, F, Ferlay, J, Soerjomataram, I, Siegel, RL, Torre, LA, Jemal, A. 2018. Global cancer statistics 2018: GLOBOCAN estimates of incidence and mortality worldwide for 36 cancers in 185 countries. CA: A Cancer Journal for Clinicians; 68(6): 394-424.

2. Monsuez, JJ, Charniot, JC, Vignat, N, Artigou, JY. 2010. Cardiac side-effects of cancer chemotherapy. International Journal of Cardiology; 144(1): 3-15.

3. Love, RR, Leventhal, H, Easterling, DV. Nerenz, DR. 1989. Side effects and emotional distress during cancer chemotherapy. Cancer; 63(3): 604-612. 
4. Oun, R, Moussa, YE. Wheate, NJ. 2018. The side effects of platinum-based chemotherapy drugs: a review for chemists. Dalton Transactions; 47(19): 6645-6653.

5. Cheok, CF. 2012. Protecting normal cells from the cytotoxicity of chemotherapy. Cell Cycle; 11(12): 2227-2227.

6. Saloustros, E, Mavroudis, D, Georgoulias, V. 2008. Paclitaxel and docetaxel in the treatment of breast cancer. Expert Opinion on Pharmacotherapy; 9(15): 2603-2616.

7. Saloustros, E, Georgoulias, V. 2008. Docetaxel in the treatment of advanced nonsmall-cell lung cancer. Expert Review of Anticancer Therapy; 8(8): 1207-1222.

8. Yin, H, Guo, R, Xu, Y, Zheng, Y, Hou, Z, Dai, X, Zhang, Z, Zheng, D, Xu, HE. 2012. Synergistic antitumor efficiency of docetaxel and curcumin against lung cancer. Acta Biochimica et Biophysica Sinica; 44(2): 147-153.

9. Baker, SD, Verweij, J, Cusatis, GA, van Schaik, RH, Marsh, S, Orwick, SJ, Franke, RM, Hu, S, Schuetz, EG, Lamba, V, Messersmith, WA, Wolff, AC, Carducci, MA, Sparreboom, A. 2009. Pharmacogenetic pathway analysis of docetaxel elimination. Clinical Pharmacology \& Therapeutics; 85(2): 155-163.

10. Takimoto, T., Nakabori, T., Osa, A., Morita, S., Terada, H., Oseto, S., Iwazawa, T. Abe, K., 2012. Tubular nephrotoxicity induced by docetaxel in non-small-cell lung cancer patients. International Journal of Clinical Oncology; 17(4): 395-398.

11. Pabla, N, Dong, Z. 2008. Cisplatin nephrotoxicity: mechanisms and renoprotective strategies. Kidney International; 73(9): 994-1007.

12. Aggarwal, BB, Kumar, A, Bharti, AC. 2003. Anticancer potential of curcumin: preclinical and clinical studies. Anticancer Research; 23(1/A): 363-398.

13. Basnet, $P$, Skalko, BN. 2011. Curcumin: An anti-inflammatory molecule from a curry spice on the path to cancer treatment. Molecules; 16(6): 4567-4598.

14. Araujo, C, Leon, L. 2001. Biological activities of Curcuma longa L. Memórias do Instituto Oswaldo Cruz; 96(5): 723-28.

15. Joe, B, Vijaykumar, M, Lokesh, B. 2004. Biological properties of curcumin-cellular and molecular mechanisms of action. Critical Reviews in Food Science and Nutrition; 44(2): 97-111.

16. Gupta, SC, Patchva, S, Koh, W, Aggarwal, BB. 2012. Discovery of curcumin, a component of golden spice, and its miraculous biological activities. Clinical and Experimental Pharmacology and Physiology; 39(3): 283-299.

17. Farombi, E, Ekor, M. 2006. Curcumin attenuates gentamicininduced renal oxidative damage in rats. Food and Chemical Toxicology; 44(9): 1443-1448.

18. Venkatanarayana, G, Sudhakara, G, Sivajyothi, P, Indira, P. 2012. Protective effects of curcumin and vitamin $\mathrm{E}$ on carbon tetrachlorideinduced nephrotoxicity in rats. EXCLI Journal; 11: 641-650.

19. Tung, BT, Hai, NT, Son, PK. 2017. Hepatoprotective effect of Phytosome Curcumin against paracetamol-induced liver toxicity in mice. Brazilian Journal of Pharmaceutical Sciences, 53(1): 1-13.

20. Berridge, MV, Herst, PM, Tan, AS. 2005. Tetrazolium dyes as tools in cell biology: New insights into their cellular reduction. Biotechnology Annual Review; 11: 127-152.
21. Rao, DK., Liu, H, Ambudkar, SV. Mayer, M. 2014. A combination of curcumin with either gramicidin or ouabain selectively kills cells that express the multidrug resistance-linked ABCG2 transporter. Journal of Biological Chemistry, 289(45): 31397-31410.

22. Adahoun, MAA, Al-Akhras, MAH, Jaafar, MS, Bououdina, M. 2017. Enhanced anti-cancer and antimicrobial activities of curcumin nanoparticles. Artificial cells, Nanomedicine, and Biotechnology; 45(1): 98-107.

23. Nishida, M, Nishiumi, S, Mizushina, Y, Fujishima, Y, Yamamoto, K, Masuda, A, Mizuno, S, Fujita, T, Morita, Y, Kutsumi, H, Yoshida, H. 2010. Monoacetylcurcumin strongly regulates inflammatory responses through inhibition of NF- $\mathrm{KB}$ activation. International Journal of Molecular Medicine; 25(5): 761-767.

24. Zhao, Y, Collier, JJ, Huang, EC. Whelan, J. 2014. Turmeric and Chinese goldthread synergistically inhibit prostate cancer cell proliferation and NF-kB signaling. Functional Foods in Health and Disease; 4(7): 312-339.

25. Ortega-Domínguez, B, Aparicio-Trejo, OE, García-Arroyo, FE, León-Contreras, JC, Tapia, E, Molina-Jijón, E, Hernández-Pandoc, R, Sánchez-Lozada, LG, Barrera-Oviedo, D, Pedraza-Chaverri, J. 2017. Curcumin prevents cisplatin-induced renal alterations in mitochondrial bioenergetics and dynamic. Food and Chemical Toxicology; 107: 373385

26. Zhou, QM, Wang, XF, Liu, XJ, Zhang, H, Lu, YY, Huang, S, Su, SB. 2011. Curcumin improves MMC-based chemotherapy by simultaneously sensitising cancer cells to MMC and reducing MMCassociated side-effects. European Journal of Cancer; 47(14): 22402247.

27. Ramanathan, B, Jan, KY, Chen, CH, Hour, TC, Yu, HJ, Pu, YS. 2005. Resistance to paclitaxel is proportional to cellular total antioxidant capacity. Cancer Research; 65(18): 8455-8460.

28. Trujillo, J, Chirino, YI, Molina-Jijón, E, Andérica-Romero, AC, Tapia, E, Pedraza-Chaverrí, J. 2013. Renoprotective effect of the antioxidant curcumin: Recent findings. Redox Biology; 1(1): 448-456.

29. Swamy, AV, Gulliaya, S, Thippeswamy, A, Koti, BC, Manjula, DV. 2012. Cardioprotective effect of curcumin against doxorubicininduced myocardial toxicity in albino rats. Indian Journal of Pharmacology; 44(1): 73.

30. Sheu, MT, Jhan, HJ, Hsieh, CM, Wang, CJ, Ho, HO. 2015. Efficacy of antioxidants as a complementary and alternative medicine (CAM) in combination with the chemotherapeutic agent doxorubicin. Integrative Cancer Therapies; 14(2): 184-195.

31. Kim, SJ, Kim, HS, Seo, YR. 2019. Understanding of ROSInducing Strategy in Anticancer Therapy. Oxidative Medicine and Cellular Longevity; 1-12.

32. Florentin, A, Arama, E. 2012. Caspase levels and execution efficiencies determine the apoptotic potential of the cell. Journal of Cell Biology; 196(4); 513-527.

33. Benzer, F, Kandemir, FM, Kucukler, S, Comakl1, S, Caglayan, C. 2018. Chemoprotective effects of curcumin on doxorubicin-induced nephrotoxicity in wistar rats: by modulating inflammatory cytokines, apoptosis, oxidative stress and oxidative DNA damage. Archives of Physiology and Biochemistry; 124(5): 448-457.

34. Dai, C, Ciccotosto, GD, Cappai, R, Tang, S, Li, D, Xie, S, Xiao, $\mathrm{X}$, Velkov, T. 2018. Curcumin attenuates colistin-induced neurotoxicity in N2a cells via anti-inflammatory activity, suppression of oxidative stress, and apoptosis. Molecular Neurobiology; 55(1): 421-434. 